\title{
High Resolution EELS in the IBM Sub-Angstrom STEM
}

P.E. Batson

IBM T.J. Watson Research Center, Yorktown Heights, New York 10598

For many years, EELS in the STEM has been used to explore nanometer-sized metal and semiconductor particles, both singly and in systems. These studies showed that the EELS response depended on many parameters, including particle and particle coating compositions, scattering impact parameter, orientation of the particles, and inter-particle spacings. It is interesting to ask whether this behavior extends down to atomic dimensions. Do groups of atoms exhibit phenomena that flow smoothly from nanometer scale objects? A related question arises in considering point and line defects in the bulk of materials. Is the EELS scattering at a dislocation sensitive to the orientation and structure of the core? These and other questions require both a very small electron probe which can be positioned accurately relative to an atomic sized object and high resolution spectroscopy to obtain EELS data at the 100-200 meV level. Aberration correction in electron microscopy holds exciting promise to address these questions.

At IBM, the first Nion corrector was installed in the VG Microscopes HB501 STEM in 2001. $[1,2]$ This system includes a high resolution Wien Filter spectrometer [3] in order to study electronic structure in semiconductors. [4] As described in a previous abstract [5], addition of the corrector required a redesign of the electron optics that couples the specimen scattering into the spectrometer to increase the collection efficiency of the spectrometer system, so that high resolution EELS measurements would be possible with the $0.8 \AA$ sized probe made available by abberation correction. In addition, a monochromator prototype operation was demonstrated before installation of the Nion aberration corrector, [6] but it has not been shown yet that it will be possible to efficiently couple this device into the aberration corrected column.

Fig. 1 shows the first zero loss obtained with the new optics. The energy distribution is dominated by the intrinsic shape of the room temperature field emission profile as in the past. This spectrum is acquired using a ccd detector in a somewhat unusual way. In order to preserve the energy calibration, the spectrum is scanned across the full ccd, treating the ccd as a set of many energy selecting slits, producing several hundred separate spectra, which are then shifted to a common energy axis and added up for the final result. This converts positional energy errors into an integrated resolution, and normalizes ccd channel to channel gain variations. Fig. 1 shows that the zero loss can be fitted with a theoretical field emission profile convoluted with a $130 \mathrm{meV}$ Gaussian distribution. This blurring is due to 2nd order aberration in this uncorrected filter and to the non-linear dispersion of the spectrum across the detector, which has not been corrected in this example. When the monochromator is reintroduced to the system, this non-linear dispersion will need correction at the detector to realize the $60-70 \mathrm{meV}$ resolution capability of the spectrometer. 
Fig. 2 shows results for the Si $2 \mathrm{p}_{3 / 2} \rightarrow$ CB scattering using the small probe optics and a beam current of $150 \mathrm{pA}$, compared with results using the system before aberration correction from [4]. The two results are nearly the same, with the onset of $\Delta_{1}$ at $99.84 \mathrm{eV}$. This spectrum is the sum of four separate acquisitions, equivalent to a single CCD integration time of about 32 sec. It shows a noise content which is about twice that obtained in the earlier result.

Currently, this system is being utilized for imaging and local analysis of hi- $\kappa \mathrm{HfO}_{2}$ dielectric structures to better understand the distribution of elements within a 6-7 $\AA$ thick $\mathrm{SiO}_{x}$ layer which lies between the $\mathrm{HfO}_{2}$ and the underlying $\mathrm{Si}$ substrate. This information has been inaccessible in conventional instruments because probe tails produce strong scattering from the nearby high atomic number material, interfering with interpretation in the oxide. Low loss scattering from this layer is also available, and contains unusual structure which may contain information about the dielectric properties of that layer.

[1] P.E. Batson, Niklas Dellby, and O.L. Krivanek, Nature, 418 (2002)617 - 620.

[2] N. Dellby, O.L. Krivanek, P.D. Nellist, P.E. Batson, and A.R. Lupini, J. Electron Microscopy, 50 (2001)177 $-185$.

[3] P.E. Batson, Rev. Sci. Inst., 57 (1986)43-48.

[4] P.E. Batson, J. Electron Microscopy, 45 (1996)51-58.

[5] P.E. Batson, in Microscopy and Microanalysis, ed. D. Piston, J. Bruley, I.M. Anderson, P. Kotula, G. Solorzano, A. Lockey, and S. McKernan (Cambridge University Press, Cambridge, 2003), pp. 836 - 837.

[6] P.E. Batson, H.W. Mook, and P. Kruit, in International Union of Microbeam Analysis 2000, ed. D.B. Williams and R. Shimizu (Institute of Physics, Bristol, 2000), Vol. 165, pp. 213 - 214.

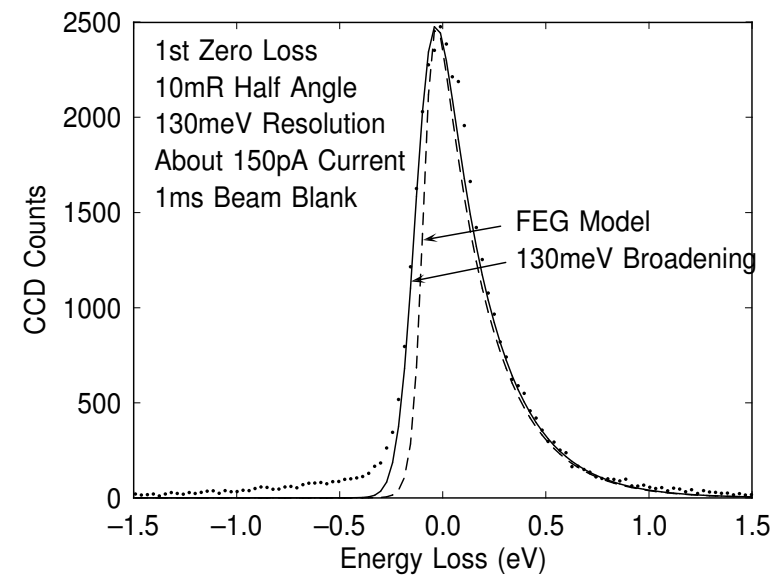

Fig. 1. First zero loss obtained with a $0.8 \AA$ probe using the new coupling optics. The total spectrum acquisition time is of order a few seconds controlled by the scan rate. The actual integration time is limited by beam blanking to about $1 \mathrm{~ms}$ per point to prevent saturation of the detector.

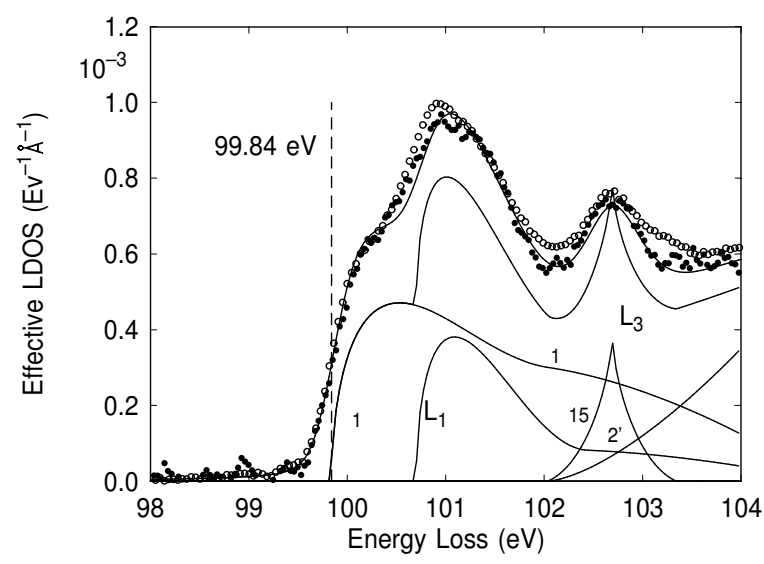

Fig. 2. $\mathrm{Si}_{3}\left(2 \mathrm{p}_{3 / 2}\right)$ edge shape obtained with the small probe optics (solid circles), compared with a result using the $2 \AA$ probe available before aberration correction (open circles).(ref [4]) Solid lines summarize a decomposition of measured scattering into final state components at Brillouin Zone positions. 